\title{
La investigación sobre historias de vida: de la identidad humanista a la subjetividad nómada
}

\author{
Research on life histories: from humanist identity \\ to nomadic subjectivity
}

Fernando Hernández-Hernández, * Juana María Sancho Gil**

Recibido: 17 de junio de 2020 Aceptado: 11 de septiembre de 2020 Publicado: 30 de septiembre de 2020

To cite this article: Hernández-Hernández, F. y Sancho, J. M (2020). La investigación sobre historias de vida: de la identidad humanista a la subjetividad nómada. Márgenes, Revista de Educación de la Universidad de Málaga, 1 (3), 34-45 DOI: https://doi.org/10.24310/mgnmar.v1i3.9609

\section{RESUMEN}

Este artículo aborda, desde la trayectoria investigadora de los autores y en diálogo con las aportaciones posthumanistas, el tema de la subjetividad en la investigación sobre historias de vida. Lo hace a partir de plantear cómo la perspectiva onto-epistemológica humanista guía buena parte de la investigación cualitativa de los años ochenta y noventa del pasado siglo. Esta orientación humanista, que afecta a cómo se plantea la investigación sobre historias de vida, coloca a la identidad de con quienes se investiga en una trama de dicotomías y binarismos. Esta trama, que resulta favorecedora de verdades y valores universales, se centra en el cogito cartesiano y en el supuesto de un sujeto investigador/investigado que sabe de sí. En la segunda parte del artículo planteamos un desplazamiento hacia una subjetividad posthumanista que presta atención a lo que sería una geografía de los ensamblajes. Este enfoque puede contribuir a que la investigación en historias de vida se lleve a cabo desde estrategias de montaje que hagan visible no la trayectoria de un sujeto singular, fijo y predecible, sino de los desplazamientos de un sujeto nómada y atravesado por multiplicidades. Con ello se trata de contribuir a una perspectiva de investigación que reconozca las limitaciones de "un” relato, que teorice la ética de las narraciones y que afronte los límites del “yo” narrativo.

Palabras clave: posthumanismo; nuevos materialismos; nuevos empirismos, subjetividades; nomadismo

\section{ABSTRACT}

From the research trajectory of the authors and in dialogue with post-humanist contributions, this article addresses the issue of subjectivity in research on life stories. It does so by considering how the humanist ontoepistemological perspective guides a large part of the qualitative research of the 1980s and 1990s. This humanist orientation, which affects how life history research is approached, places the identity of those being researched in a web of dichotomies and binarisms. This plot, which is conducive to universal truths and values, focuses on the Cartesian cogito and the assumption of the researcher/researched who knows about themselves. In the second part we propose a shift towards a post-humanist subjectivity that pays attention to what would be a geography of assemblages. This approach can contribute to research into life histories from assembly strategies that make 
visible not the trajectory of a singular, fixed, and predictable subject, but the displacements of a nomadic subject crossed by multiplicities. As this is about contributing to a research perspective that recognises the limitations of 'one' story, that theorises the ethics of narratives and that confronts the limits of the narrative 'I'.

Keywords: posthumanism; new materialisms; new empiricism, subjectivities; nomadism

\section{INTRODUCCIÓN: Nuestra trayectoria por las historias de viday la cuestión de laidentidad como constructo humanista}

A estas alturas de nuestra trayectoria investigadora de casi 20 años en historias de vida, nos parece necesario poner en relación lo que hemos aprendido en este campo con la cuestión que abordamos en este artículo: la necesidad de problematizar el fundamento humanista de esta perspectiva de investigación. Entramos en la investigación narrativa como perspectiva ontoepistemología y ética interpretativa en los inicios la década del milenio, influidos por el giro al yo (Hernández y Rifá, 2011) que recorría las ciencias sociales durante la década de 1990. Este giro configuró una perspectiva de investigación narrativa, considerada como una modalidad de investigación cualitativa que, en un sentido amplio, se basaba en narrativas que describían la acción humana (Casey, 1995-96). El término 'investigación narrativa' tuvo su carácter fundacional a partir del artículo que Conelly \& Clandinin (1990) publican en Educational Researcher, en el que destacan una doble influencia que se relacionaba con el cuestionamiento -por su insuficienciadel paradigma positivista/realista a la hora de captar y dar cuenta de las experiencias de vida de los seres humanos, y con la deriva que adopta la investigación en ciencias sociales hacia una investigación que recoja y dé sentido a esas experiencias. Este giro dio paso a una fecunda línea de investigación (Clandinin \&Conelly, 1995), que se articula en torno a lo que Denzin (1997) denominó narrativas del yo, en la que quien investiga forma parte y aparece en un hilo narrativo, que no se impone al lector, sino que le deja lugar, para que establezca sus propios nexos y relaciones (Conelly \& Clandinin, 2000). Este movimiento permitía “alterar y cuestionar las normas del discurso científico que se consideran incuestionables a partir de destacar la experiencia vivida, los detalles íntimos, la subjetividad y las perspectivas personales” (Ellingson \& Ellis, 2008, p. 450). En este marco, las investigaciones que tienen que ver con las historias de vida, especialmente de docentes, se vinculan con el interés de la academia por las situaciones, procesos, experiencias y posicionamientos vinculados, sobre todo, a cómo el profesorado construye y negocia su identidad, y a cómo aprende a ser docente (Maclure, 1993; Clandinin \& Connelly, 1995; Goodson \& Hargreaves, 1996; Gee, 2001; Goodson, 2003, 2004; Sfard \& Prusak, 2005; Flores \& Day, 2006).

Fue en este contexto en el que llevamos a cabo las investigaciones "Análisis del impacto de los cambios sociales y profesionales en el trabajo y la vida de los docentes" (Sancho, 2011) y "Los efectos de los cambios sociales en la vida profesional de los docentes universitarios" (Sancho, 2013). Y, en particular con nuestra participación en el proyecto europeo PROFKNOW-Professional Knowledge in Education And Health: Restructuring work and life between the state and the citizens in Europe (Müller et al., 2008, 2011), en el que nos planteamos, a partir de historias de vida de docentes y enfermeras, comprender cómo los procesos de reestructuración que por entonces se afianzaban en países como Reino Unido y Estados Unidos, y que algunos años más tarde llegaron a otros países como España, estaban afectando a profesiones del cuidado en en- 


\section{E S T U D I O Y E N SAYOS}

fermería y magisterio. Esta investigación, realizada desde una perspectiva sociológica, las historias de vida se llevan a cabo no para dar cuenta de procesos individuales, sino para hacer visibles, a través de ellos, aspectos de la vida social, en este caso relacionados con la reestructuración profesional. Este estudio, seguía la divisa de Bourdieu (1990), quien plantea que los investigadores sociales deben procurar llevar los conceptos o categorías de análisis, que son productos del mundo social al que ellos pertenecen, a la realidad social que estudian. Lo que nos llevó a realizar historias de vida, en la línea propuesta por Goodson \& Hargreaves (1996) y Goodson $(2003,2004)$ quienes focalizan las historias en los conceptos previamente establecidos. Este último autor fue uno de los coordinadores del proyecto Profknow. Investigación en la que, cuando comenzamos a realizar el análisis de las entrevistas, se nos plantearon dilemas relacionados con el ejercicio del poder del investigador, con su proyección teórica sobre las experiencias de los sujetos y con la tentación de establecer una posición interpretativa del yo.

Con este bagaje nos acercarnos a la experiencia de jóvenes considerados como 'fracasados' para el sistema escolar (Hernández, 2011a). Continuamos recogiendo y construyendo historias de jóvenes en su relación con el saber en la escuela secundaria (Hernández, 2011b) y de jóvenes emigrantes de éxito académico (Sancho et al., 2012). Como hemos señalado, también prestamos atención a los docentes universitarios (Sancho, 2013) para tratar de comprender cómo experimentaban el impacto del cambio económico, social, cultural, tecnológico y laboral que afectaba a las universidades españolas, así como a sus vidas e identidades profesionales. También fueron las historias de vida las que nos permitieron acercarnos a cómo las maestras de primaria transitaban por su periodo de formación y los primeros años de su trayectoria profesional (Sancho Gil y Hernández-Hernández, 2014). En esta última investigación nos planteamos la necesidad de describir, analizar e interpretar las nociones, representaciones y experiencias relacionadas con la constitución de la subjetividad profesional de los maestros y maestras de educación infantil y primaria, especialmente durante la formación inicial y a los primeros años de trabajo en la escuela.

Todos estos estudios nos desvelaron la importancia de las experiencias biográficas, recogidas y articuladas en historias de vida, que daban cuenta de las trayectorias escolares y profesionales. Este recorrido nos llevó a reflexionar sobre qué aproximación a la subjetividad (como forma de conceptualizar el relato de vida de los sujetos) proyectábamos los investigadores en las historias de vida que construimos. Además de sobre los marcos onto-epistemológicos, metodológicos y éticos en los que fundamentamos las decisiones investigadoras. Como esta es una reflexión que viene de lejos, hemos dejado huella de la misma, tanto en artículos sobre historias de vida (Hernández-Hernández y Sancho-Gil, 2018), como en relación a la posicionalidad del investigador (Hernández-Hernández y Revelles Benavente, 2019; Hernández, Aberasturi, Sancho y Correa, 2020) Algunas de estas aportaciones vuelven a aparecer en este texto, pues uno nunca parte de cero, para sustentar el desplazamiento, la novedad, si puede hablarse de esta manera, que aquí presentamos: el tránsito de una identidad humanista a una subjetividad posthumanista en la investigación cualitativa en general, y en las historias de vida, en particular

Antes de continuar es necesario una aclaración. A lo largo del texto optamos por la noción de subjetividad. Pero en la genealogía de la investigación narrativa y de las historias de vida, el concepto que se configuró durante tiempo como central fue el de identidad (personal, docente, profesional). Noción que deriva en buena medida de los trabajos de Erikson en los años 1950s 


\section{EST U D O S Y ENSAYOS}

(Erikson, 1968). Guzmán Palacios (2017), en la revisión que realiza del término, señala dos corrientes principales a partir de la mitad de los años setenta del pasado siglo. La primera desde los estudios de Epstein (1978) quien, desde la antropología, caracteriza la identidad (individual y colectiva) como el proceso por el cual una persona intenta integrar sus diversos status y roles en una imagen coherente del yo. La segunda, a partir de los trabajos de Ehle (1989), se relaciona con las categorías de autoconcepto, autoestima, autoideal y autoconfianza, y pone el foco en cómo un sujeto se evalúa a sí mismo (autoestima), cómo desea ser (ideal de sí mismo) y lo seguro que está de sí mismo (autoconfianza). El elemento común entre ambas perspectivas, y que puede vincularse con las historias de vida, relaciona la identidad con lo que los individuos dicen sobre qué y cómo son y sobre cómo se relacionan con los demás (Gee, 2001). Los estudios sobre la identidad reconocen que las identidades no son constructos aislados sino co-construcciones entre el individuo, sus entornos (surroundings) y sus relaciones. Por lo tanto, la identidad se focaliza en los individuos, sus acciones y su 'agency' -capacidad de acción, capacidad de ser- (Shanahan, 2009). Como se ha señalado en Hernández (2019) esta visión de la identidad -y la investigación sobre historias de vida que trata de reflejarla- se vincula a una investigación interpretativa que Lather (2013) denomina como convencional y que surge de una visión humanista que proyecta un sujeto al que se le supone que tiene una voz auténtica y genuina. Esa voz y las acciones de ese sujeto son las que le posibilitan realizar descripciones transparentes de las experiencias vividas, al tiempo que se asume que los métodos que facilitan esas descripciones pueden acercarnos a la 'verdad' de la experiencia de los sujetos.

Cuando nos comenzamos a acercar en las historias de vida a la cuestión de la identidad relacionada con los docentes, se tendía a perfilarla como un referente de características estables e íntegras, proyectando una visión moderna del sentido de ser que, ya por entonces cuestionada desde posiciones construccionistas (Gergen, 1991; Grodin \& Lindlof, 1996). El reflejo de esta aproximación se puede encontrar, si se revisan las aportaciones presentadas a las diferentes jornadas sobre Historias de Vida en Educación organizadas desde diferentes universidades españolas (Hernández, Sancho y Rivas, 2011; Rivas, Hernández, Sancho y Núñez, 2012; Lopes, Hernández, Sancho y Rivas, 2013). En las contribuciones se aprecia, como preocupaciones principales: la búsqueda de métodos de análisis - por lo general tematizaciones- de las transcripciones de las entrevistas con los colaboradores; el tránsito entre los relatos de vida, que ponen en énfasis en el carácter reconstructivo de la narración individual de la experiencia (life stories) y las historias de vida (life histories) en las que el interés está en aquello que lo individual nos permite comprender de la vida social; la profundización en el sentido y el valor de la 'experiencia' como evidencia para la investigación; y la búsqueda de relaciones -a veces teleológicas-entre el pasado y el presente de los docentes. Con la perspectiva de casi dos décadas, y conscientes de que estas prioridades lo eran también para la comunidad investigadora de entonces, como hemos apuntado en Hernández (2019), hoy consideramos que estos enfoques de las historias de vida quizá no tenían en cuenta que poner la centralidad en la voz y la experiencia de los sujetos plantea problemas relacionados con el sentido androcéntrico de la subjetividad y la experiencia, la interpretación que de ella realiza quien investiga y la ontología y epistemología humanista que lo guía. Lo que hace necesario, como señala Biesta (2013) evitar "ciertas otras palabras y conceptos, en particular la noción de identidad, que tiene más que ver con las formas en que nos identificamos dentro de los órdenes y tradiciones existentes que con las formas de actuar y ser que están "fuera” de esto" (p. 18, énfasis original). (Traducción de los autores). 


\section{EST U D O S Y E N A Y O S}

Quizá por todo lo anterior, Jackson y Mazzei (2011) recogen -en relación con la investigación autoetnografía- algunos cuestionamientos que también se pueden atribuir a las historias de vida de docentes: ser poco analíticas y autoindulgentes, ensimismadas, sentimentales y románticas. Nosotros, como han hecho las autoetnógrafas, podríamos responder a estas críticas diciendo que son formas más encarnadas (Sparkes, 2007), políticas (Holman-Jones, 2005), veraces (Ellis, 2001; Ellis y Bochner, 2000), experimentales (Bochner y\& Ellis, 1996) y reflexivas (Ellis \& Bochner, 2000) de hacer ciencia social. Sin embargo, estas críticas las consideramos una oportunidad para participar en la reflexión en torno a la necesidad de que las historias de vida "anhelen ser utilizadas en lugar de analizadas; ser contadas y recontadas en lugar de teorizadas y establecidas” (Ellis \& Bochner, 2000, p. 744). Como ellas, tratamos de buscar una narración que reconozca las limitaciones de "un" relato, que teorice la ética de tales relatos, que trabaje los límites del "yo" narrativo y que se plantee, como señalan Alicia Jackson y Lisa Mazzei (2011) que la cuestión no se centra en qué 'es' la investigación autoetnográfica (en historias de vida) sino en lo que se pretende 'hacer' en y con ella.

Porque estas cuestiones nos afectan, en la investigación sobre las historias de vida de docentes que se encontraban en los cinco primeros años de ejercicio profesional (Sancho Gil y Hernández Hernández, 2014), comenzamos a revisar la noción de identidad docente en cuanto constructo. Un constructo que deviene de una trama de permanentes interacciones de los individuos -cada uno con su bagaje biológico, biográfico y social-, con las políticas educativas, los compañeros, las estructuras y relaciones de poder de los centros, el alumnado y las familias. Esta concepción no fija de la identidad, se vincula, como señala Lather (2013), con una perspectiva de la investigación cualitativa que comienza a reconocer múltiples realidades, voces, textos que se recogen y presentan de maneras desordenadas. Donde la centralidad ya no está en la identidad de quien da cuenta de su experiencia, sino de las relaciones que la posibilitan. Lo que lleva a introducir nociones como reflexividad, diálogo, empoderamiento, etc., que permanecen dentro del marco onto-epistemológico humanista, que se fundamenta en los conceptos humanistas de lenguaje, realidad, conocimiento, poder, verdad, resistencia y sujeto. Esta investigación nos abrió el camino hacia una revisión de la noción de identidad y de la investigación humanista que nos había guiado en nuestra aproximación a las historias de vida. Revisión que comenzó a articularse cuando, en el contexto del proyecto de investigación sobre cómo aprenden los docentes (Hernández, Aberasturi, Sancho y Correa, 2020) nos acercamos al giro postcualitativo (HernándezHernández y Revelles Benavente, 2019). Lo que nos ha llevado a considerar el sentido de lo que puede ser investigar desde otras coordenadas onto-epistemológicas, metodológicas y éticas, y desde una noción de subjetividad de la que damos cuenta en el siguiente apartado.

\section{CONCEPTUALIZACIÓN:LASUBJETIVIDADDESDEUNAPERSPECTIVAPOSTHUMANISTA}

Smithers y Eaton, 2017 señalan que una limitación de la teorización sobre la identidad y la subjetividad desde perspectivas psicológicas y postestructuralistas es la indexación negativa y las limitaciones que se imponen a las posibilidades para el devenir humano (y no humano). Para esquivar este condicionamiento hemos tomado las aportaciones de la perspectiva posthumanista, y en particular las referencias a la subjetividad nómada de Rossi Braidotti. 


\section{E S T U D I O Y E N S Y O S}

Como hemos recogido en Hernández-Hernández (en prensa), Snaza et al., (2014) señalan que el significado del ser humano en relación con las tecnologías, los animales y los objetos es inseparable de la necesidad de repensar los conceptos que utilizamos para entender cómo se relacionan las cosas -vivas y no vivas-, especialmente en la política y el conocimiento. En esta dirección, Braidotti (2013/2015), siguiendo el camino iniciado por Haraway (1984/1991), señala que esta relación está encarnada y arraigada en las prácticas de vida, en las condiciones históricas y los lugares en los que habitan los cuerpos. En esta ontología, la subjetividad no se diluye como en el proyecto humanista, ni se configura como un "sujeto ausente" o unificado (Deleuze y Guattari, 1972/1973). Desde esta mirada, la subjetividad posthumana reconfigura las identidades y las prácticas humanísticas de manera que producen nuevas subjetividades y formas de hacer que aún no han sido codificadas en modelos heteronormativos y/o disciplinarios (Braidotti, 2013/2015). Este planteamiento, según Braidotti, conlleva un “conceptual earthquake” que está afectando al campo de las humanidades, y que pensamos podría ayudar a la investigación en historias de vida a revisar sus fundamentos. La posición de Braidotti es que el proyecto humanista, que se conecta y deriva del legado de la Ilustración, ha puesto de manifiesto sus contradicciones relacionadas con el gobierno de las oposiciones binarias (humano-animal; humano-máquina; vivo/no vivo; naturaleza-cultura) y las relaciones de poder constituidas a partir de estas oposiciones. Una consecuencia de estas contradicciones ha sido hacer visible que el proyecto humanista era/es, en buena medida, a la vez eurocéntrico y racista. Para salir de estas contradicciones una de las nociones es la de subjetividad. Rossi Braidotti, como parte de un proyecto más contra la rigidez metodológica, el esencialismo y el universalismo, enmarca la subjetividad en una perspectiva nómada que surge en medio de las dislocaciones introducidas por las estructuras capitalistas avanzadas, la proliferación de tecnologías y las limitaciones de la teorización psicológica sobre nuestra comprensión de la identidad (Smithers \& Eaton, 2017). Braidotti plantea este sentido de la subjetividad a partir de reconocer que: "vivimos en procesos permanentes de transición, hibridación y nomadización. Y estos estados intermedios y los escenarios desafían los modos de representación teórica establecidos, precisamente porque son zigzagueante, no lineales y orientados a los procesos, no impulsados por conceptos” (Braidotti, 2011b, p. 217). El nomadismo al que se refiere Braidotti se proyecta como una posibilidad de posicionamiento del sujeto a través de una confluencia de factores indicativos de la época actual. En este sentido, la subjetividad nómada tiene sus raíces en un imperativo ético de movimiento, cambiando nuestras narrativas teleológicas de control, que denomina 'potestas' hacia el reconocimiento de la relacionalidad ética en los espacios ecológicos, cosmológicos y tecnológicos cambiantes.

Este movimiento posibilita que, en lugar de consignarnos a posiciones de sujeto o identidad particulares, la subjetividad nómada nos ofrece nuevas oportunidades para abrir el mundo a estructuras políticas radicalmente diferentes. La subjetividad nómada se abre a un mundo posthumano, reconociendo la conectividad relacional entre los humanos, otras especies, tecnologías, espacios geográficos, tiempos cronológico y aiónico (circular). Algo que resulta necesario en la comprensión de la perspectiva situada de la subjetividad comprometida. Tal enredo desplaza las discusiones de las perspectivas exclusivamente humanas (antropomórficas) e interrumpe en las nociones de subjetividad individual, dando paso a la subjetividad relacional. El pensamiento relacional, enfocado no sólo en términos de relaciones humanas (como hace el construccionismo social), hace posible un mundo que no se centra en el sujeto controlado en/dividido, sino en el 


\section{ESTUDIOS Y ENSAYOS}

potencial del desarrollo de cada momento presente. Este desplazamiento hacia la subjetividad nómada resulta clave para mover el foco de las historias de vida desde la identidad, pues considera al sujeto y la subjetividad no solo como relacional sino constituida en y por la multiplicidad y está simultáneamente encarnado y localizado, no sólo geográficamente, sino también efectivamente a lo largo de jerarquías de género, etnia y clase. Este cuestionamiento posibilita nuevos tipos de subjetividades que encontramos en los mestizajes culturales y en la actual propagación de una diversidad de géneros y sexualidades, además de las ya señaladas interconexiones entre humanos y no humanos. En consecuencia, esta subjetividad es más caótica que controlada, más distribuida que autónoma.

Este movimiento celebra las diferencias metodológicas y la complejidad de la investigación cualitativa -y las historias de vida- y aboga por una mayor apertura, imaginación y asunción de riesgos, especialmente para llevar a cabo investigaciones basadas en "post-teorías" (nuevo materialismo, nuevo empirismo, posthumanismo (ver Sancho-Gil \& Correa-Gorospe (2019), Revelles Benavente \& Sancho-Gil (2020) para una introducción a estos campos de estudio). Estas nuevas ontologías demandan desplazamientos metodológicos, afrontar incidentes, saltos conceptuales y deslizamientos, así como hacer evidentes los vínculos teóricos. En la investigación humanística cualitativa convencional (St. Pierre, 2011) -la que nos ha guiado en las historias de vida- las referencias metodológicas y los métodos que las conectan con la realidad suelen considerarse relativamente simples y concretos, en comparación con lo que sucedería si se consideraran las subjetividades de manera más compleja. Lo que podría contribuir a generar conocimientos, conceptos y argumentos de manera diferente. Este marco permite pensar el sentido de la investigación de historias de vida desde otra perspectiva, con otros fundamentos y propósitos. Pero, sobre todo, nos permite argumentar que la investigación no se limita a seguir un camino prefijado, en el que se aplican métodos, para dar cuenta de los resultados previstos en las preguntas iniciales (Hernández-Hernández, 2019, p. 27).

Las autoras citadas (Braidotti, Haraway, Lather, St. Pierre), y otras que se mueven en esta dirección (Kohler-Riessman, 2008; Jackson \& Mazzei, 2012; Revelles Benavente, 2018, entre otras) problematizan la tradición humanista que ha guiado las historias de vida al tiempo que buscan alternativas en el sentido de la invitación que realizan Lather y St. Pierre (2013):

Si dejamos de privilegiar el saber sobre el ser; si rechazamos las suposiciones positivistas y fenomenológicas sobre la naturaleza de la experiencia vivida y del mundo; si renunciamos a la lógica representacional y binaria; si vemos el lenguaje, lo humano y lo material no como entidades separadas mezcladas entre sí sino como completamente imbricadas "en la superficie" -si hacemos todo eso y cuanto más se abra- ¿será posible la investigación cualitativa tal como la conocemos? Tal vez no. (p. 630). (Traducción de los autores).

Reconocer estos 'si' supone reconocer que quien investiga siempre está enredada (entangled) en una trama que le antecede y que interviene en el proceso de la investigación. Esta situación de enredo lleva a Lather y St. Pierre (2013) a cuestionar el sentido que se le da a la investigación cualitativa, y añadimos nosotros, a algunas tendencias dominantes en las historias de vida: 


\section{E S T U D I O S Y E N S A Y O S}

El enredo hace que todas las categorías de investigación cualitativa humanista sean problemáticas. Por ejemplo, ¿cómo determinamos el “objeto de nuestro conocimiento”, el “problema” que queremos estudiar en el ensamblaje? ¿Podemos desconectarnos del enredo de alguna manera (el "Yo”) y luego desconectar cuidadosamente alguna otra pequeña parte del enredo (el "Otro") el tiempo suficiente para estudiarlo? ¿Quéontología nos ha permitido creer que el mundo es estable para que podamos hacer todo eso de la individuación? ¿Y a qué precio? ¿Cómo pensamos que un "problema de investigación” en la imbricación de un ensamblaje agéntico de diversos elementos que están constantemente intraactuando, nunca estables, nunca iguales? (p.630). (Traducción de los autores).

Según St. Pierre (2011), estos giros “anuncian una ruptura radical con los supuestos humanistas, modernistas, imperialistas, representacionistas, objetivistas, racionalistas, epistemológicos, ontológicos y metodológicos del pensamiento y la práctica de la Ilustración occidental” (p. 615). Cambios que pueden contribuir a otro sentido de la investigación en historias de vida en educación.

\section{CONCLUSIONES. UNA SUBJETIVIDAD NÓMADA EN LAS HISTORIAS DE VIDA}

La investigación en historias de vida, especialmente en el campo de la educación y relacionada con las trayectorias de docentes, supuso una aportación relevante desde el giro narrativo que contribuyó, no solo a dar valor a la experiencia, sino a considerar la complejidad de los individuos y evitar su reduccionismo a categorías simplificadores y previamente definidas. Sin embargo, y como ha sucedido con otras formas de investigación cualitativa, las historias de vida han podido caer en el metodocentrismo. Es decir, en la preocupación por el análisis y tematización de las transcripciones de las entrevistas, y por considerar a los sujetos que se narran y que colaboran en la investigación desde el prisma de una concepción humanista del sujeto, tal y como hemos señalado más arriba.

Lo que nos lleva como alternativa, y siguiendo a Braidotti (2006, 2011, 2014), a rastrear el sentido de un sujeto nómada para comprender, por ejemplo, la red de relaciones de poder que lo constituyen. Braidotti (2006) sitúa al sujeto nómada en la intersección con las fuerzas relacionales externas, para cuyo estudio se requiere afrontarla como ensamblajes. Encontrarlos es una cuestión de geografías, porque se trata de apuntar en el relato orientaciones, puntos de entrada y salida en un constante despliegue. Donde nociones como frontera, encuadre o prácticas de contención son cruciales para toda la investigación. Al desarrollar una política de localización, Braidotti sugiere que la labor del montaje es "una forma de incorporar la práctica crítica en una perspectiva específicamente situada, evitando las generalizaciones universalistas y fundamentándola para hacerla responsable” (2006, p. 79). Esto lleva, en la investigación sobre historias de vida, a estar atento, parafraseando a Fendler (2015, p. 19-20) a esas orientaciones, puntos de entrada y salida y despliegues constantes que sostienen y apoyan los trayectos de los sujetos, como práctica de investigación en torno a una subjetividad nómada.

Todo lo anterior tiene repercusiones para la investigación en historias de vida, si se tienen en cuenta las propuestas que plantea Stephanie Springgay (2015), a la hora de realizar una investigación que asuma los planteamientos desgranados en este artículo. En primer lugar, para mantener la tensión del movimiento frente el empleo de 'métodos' preestablecidos a favor de procedimientos que surgen "in the midst of the research process" (p. 81). En segundo lugar, para 


\section{E S T U D I S Y E N A Y O S}

adoptar una construcción procesual, que considera la investigación no como rígida o limitada por un método, sino como una "ongoing construction" que sólo puede conducir "a approximate rigorous abstractions” (p. 84). Algo que implica desafiar las nociones tradicionales de investigación centradas en la causalidad lineal a las que con frecuencia tiende la investigación en historias de vida. Finalmente, respondiendo a la invitación de Elizabeth St. Pierre (2013), Springgay pide a los investigadores educativos que consideren la idea de una investigación sin datos prestablecidos, fijos, concretos y aislados, pues la investigación posthumanista persigue lo que es relacional y procesual. Lo que supone un desafío para quienes transitamos en el campo de las historias de vida en educación.

\section{AGRADECIMIENTOS}

Quisiéramos agradecer a los revisores anónimos que con sus valiosos comentarios y sugerencias han hecho que mejorara la calidad y claridad de este artículo.

\section{REFERENCIAS}

Biesta, G. (2013). The Beautiful Risk of Education. Boulder, CO: Paradigm Publishers.

Bochner, A. \& Ellis, C. (2002). Ethnographically speaking: Autoethnography, literature, and aesthetics. Walnut Creek, CA: AltaMira.

Braidotti, R. (2011a). Nomadic subjects: Embodiment and sexual deference in contemporary feminist theory (2nd ed.). New York, NY: Columbia University Press.

Braidotti, R. (2011b). Nomadic theory: The portable Rosi Braidotti. New York, NY: Columbia University Press. Braidotti, R. (2014). Writing as a nomadic subject. Comparative Critical Studies, 11(2): 163-184

Braidotti, R. (2006). Transpositions: On nomadic ethics. Cambridge, UK and Malden, MA: Polity Press.

Braidotti, R. (2013/2015). Lo Posthumano. (Traducción Juan Carlos Gentile). Barcelona: Gedisa.

Casey, K. (1995-96). The new narrative research in education. Review of research in Education, 21, 211-253.

Clandinin, D. J. \& Connelly, F. M. (1995). Teachers' professional knowledge landscapes. New York: Teachers College Press.

Connelly, M. \& Clandini, J. (2000). Narrative inquiry. San Francisco: Jossey-Bass.

Connelly, M. \& Clandinin, J. (1990). Stories of experience and narrative inquiry. Educational Researcher, $19(4), 2-14$.

Deleuze, G. y Guattari, F. (1972/1973). El Anti-Edipo. Capitalismo y esquizofrenia. (Traducción Francisco Monge). Barcelona: Barral editores.

Ehle, M.J. (1989). Self-perception and learning. Education and Society, 7(1), 46-51.

Ellingson, L. \& Ellis, C. (2008). Autoethnography as Constructionist Project. En J. A. Holstein \& J. F. Gubrium (eds.). Handbook of constructionist research (pp. 445-465). New York: The Guildford Press. 


\section{E S T U D I S Y E N A Y O S}

Ellis, C. \& Bochner, A. (2000). Autoethnography, personal narrative, and reflexivity: Researcher as Subject. En N. Denzin \& Y. Lincoln (Eds.), The handbook of qualitative research (2nd ed.). Thousand Oaks, CA: Sage Publications.

Ellis, C. (2001). With mother/with Child: A true story. Qualitative Inquiry, 7(5), 598-61

Epstein, A. L. (1978). Ethos and Identity. Three Studies in Ethnicity. London: Tavistock Publications.

Erikson, E. H. (1968). Identity: Youth and Crisis. New York: Norton

Fendler, R. (2015). Navigating the eventful space of learning: Mobilities, nomadism and other tactical maneuvers. Barcelona: Universitat de Barcelona. Depòsit Digital: http://hdl.handle.net/2445/67739

Flores, M. A. \& Day, Ch. (2006). Contexts which shape and reshape new teachers' identities: A multiperspective study. Teaching and Teacher Education, 22, 219-232.

Gee, J. P. (2001). Identity as an analytical lens for research in education. Review of Research in Education, $25,99-125$.

Gergen, K.J. (1992). El yo saturado. Dilemas de identidad en el mundo contemporáneo. Barcelona: Paidós.

Goodson, I. (2003). Professional Knowledge, Professional Lives. Maidenhead, Philadelphia: Open University Press.

Goodson, I. (ed.), (2004). Historias de vida del profesorado. Barcelona: Octaedro.

Goodson, I. \& Hargreaves, A. (1996). Teachers' Professionals Lives. London: Falmer Press.

Grodin, D. \& Lindlof, T.R. (1996). Constructing the self in a mediated world. London: Sage.

Guzmán Palacios, L.A. (2017). La construcción de la identidad profesional docente. Girona: Dipòsit Digital Universitat de Girona.

Haraway, D. (1984/1991). Ciencia, cyborgs y mujeres. La reinvención de la naturaleza. (Traducción Manuel Talens). Madrid: Cátedra.

Hernández, F. (Coord.). (2011a). Cap a una escola secundaria inclusiva. Sabers i experiències de joves em sitació d'exclusió. Barcelona: Dipòsit digital de la Universitat de Barcelona. http://hdl.handle. net $/ 2445 / 15963$

Hernández, F. (Coord.). (2011b). ¿Qué nos cuentan los jóvenes? Narraciones biográficas sobre las relaciones de los jóvenes con el saber en la escuela secundaria. Barcelona: Universitat de Barcelona. Depósito digital. http://hdl.handle.net/2445/18348

Hernández-Hernández, F. (2019). Investigar en educación desde una posición de no saber: dar cuenta de los tránsitos de una investigación en torno a cómo aprende el profesorado de secundaria. Investigación en la Escuela. Revista internacional de investigación e innovación educativa, 99: 1-13. http://dx.doi.org/10.12795/IE.2019.199.01

Hernández, F. y Rifà, M. (coords.) (2011). Investigación autobiográfica y cambio social. Barcelona: Octaedro.

Hernández, F., Sancho,J.M. y Rivas, J.I. (coord.) (2011). Historias de vida en educación: Biografías en contexto. Barcelona. Universitat de Barcelona. Depósito digital: http://hdl.handle.net/2445/15323

Hernández-Hernández, F. \& Sancho-Gil,J.M. (2018). Historias de vida y narrativas sobrela subalternidad: Afrontar el desafío de lo inabordable de la relación con el Otro. Educar, 54(1), 1-15. http://hdl.handle. net/10.5565/rev/educar.913 


\section{E S T U D I S Y E N A Y O S}

Hernández-Hernández, F. y Revelles Benavente, B. (2019). La perspectiva post-cualitativa en la investigación educativa: genealogía, movimientos, posibilidades y tensiones, Educatio Siglo XXI, 37(2), 21-48. http://doi.org/10.6018/i/387001

Hernández, F., Aberasturi, E., Sancho, J. M. y Correa, J. M. (coords.), (2020). ¿Cómo aprenden los docentes? Tránsitos entre cartografías, experiencias, corporeidades y afectos. Barcelona: Octaedro.

Hernández-Hernández, F. (en prensa). El posthumanismo como apertura a otros modos de conocer: pensar y plantear una educación y una investigación posthumana. En R. Huerta y A. Alonso Sanz (coords.) Humanidades digitales y pedagogías culturales: Saberes virales para una nueva educación. Barcelona: Ediciones UOC.

Holman-Jones, S. (2005). Auto Ethnography: Making the Personal Political. En N.K Denzin \& Y.S. Lincoln (Eds.), Handbook of Qualitative Research (pp-763-791), Sage, Thousand Oaks, https://dugi-doc.udg. edu/bitstream/handle/10256/15418/tlagp_20180228.pdf?sequence=1\&isAllowed=y

Jackson, A. Y. \& Mazzei, L. A. (2012). Thinking with Theory in Qualitative Research. London, New York: Routledge.

Jackson, A.Y. \& Mazzei, L.A. (2008). Experience and "I" in Autoethnography. International Review of Qualitative Research, 1 (3), 299-3.http://dx.doi.org/10.13140/RG.2.1.3282.4800

Kohler-Riessman, C. (2008). Narrative methods for the human sciences. Thousand Oaks, CA: Sage.

Koro-Ljungberg, M. (2015). Reconceptualizing qualitative research. Methodologies without Methodology. London: Sage.

Lather, P. (2013). Methodology-21: what do we do in the afterward? International Journal of Qualitative Studies in Education, 26:6, 634-645. http://dx.doi.org/10.1080/09518398.2013.788753

Lather, P. \& St. Pierre, E. A. (2013). Introduction: Post-qualitative research. International Journal of Qualitative Studies in Education, 26(6), 629-633.http://dx.doi.org/10.1080/09518398.2013.788752

Lopes, A., Hernández, F., Sancho, J.M. y Rivas, J.I. (coord.) (2013). Histórias de Vida em Educação: a Construção do Conhecimento a partir de Histórias de Vida. Barcelona: Universitat de Barcelona. Dipòsit Digital: http://hdl.handle.net/2445/47252

Maclure, M. (1993). Arguing for yourself: Identity as an organising principle in teachers' jobs and lives. British Educational Research Journal, 19(4), 311-322.

Revelles-Benavente, B. y Sancho-Gil, J.M. (2020). Re-explorando la "materia”: Implicaciones para la investigación educativa y social. En J.M. Sancho Gil, F. Hernández, L. Montero Mesa, J. Pablos Pons, I. Rivas-Flores, \& A. Ocaña Fernández(coords.). Caminos y derivas para otra investigación educativa y social. (pp. 37-50). Barcelona: Octaedro.

Rivas, J. I., Hernández, F., Sancho, J. M. y Núñez, C. (coord.) (2012). Historias de vida en educación: Sujeto, Diálogo, Experiencia. Barcelona: Dipòsit Digital UB. http://hdl.handle.net/2445/32345

Sancho, J.M. (Coord.) (2011). Con voz propia. Los cambios sociales y profesionales desde la experiencia de los docentes. Barcelona: Octaedro.

Sancho, J, M. (Coord.) (2013). Trayectorias docentes e investigadoras en la universidad. 24 historias de vida profesional. Barcelona: Dipòsit digital de la Universitat de Barcelona. http://diposit.ub.edu/ dspace/handle/2445/44965 


\section{EST U D I S Y E N S Y OS}

Sancho, J. M., Correa, J. M., Giró, X. y Fraga, L. (Coord.) (2014). Aprender a ser docente en un mundo en cambio. Simposio internacional. Barcelona: Dipòsit Digital de la Universitat de Barcelona. http:// hdl.handle.net $/ 2445 / 50680$

Sancho, J.M. y Hernández-Hernández, F. (2014). Maestros al vaivén. Aprender la profesión docente en el mundo actual. Barcelona: Octaedro.

Sancho, J.M., Hernández-Hernández, F., Herraiz, F., Padilla Petry, P., Fendler, R., Arrazola, A., Giró, G. y Valenzuela. R. (2012). Memòria del projecte: Trajectòries d'èxit de joves immigrants a l'ensenyament superior i al món profesional. Barcelona: Universitat de Barcelona. Depósito digital. http://hdl. handle.net/2445/32672

Sancho-Gil, J.M. y Correa-Gorospe, J.M. (2019). Intra-acciones en el aprender de docentes de infantil, primaria y secundaria Educatio Siglo XXI, 37, 115-140. http://doi.org/10.6018/educatio.387041

Sfard, A. \& Prusak, A. (2005). Telling Identities: In Search of an Analytic Tool for Investigating Learning as a Culturally Shaped Activity. Educational Researcher, 34(4), 14-22.

Shanahan, M-C. (2009). Identity in science learning: exploring the attention given to agency and structure in studies of identity, Studies in Science Education, 45(1), 43-64, http://doi. $\operatorname{org} / \underline{10.1080 / 03057260802681847}$

Smithers, L.E. \& Eaton, P. W. (2017). Nomadic Subjectivity: Movement in Contemporary Student Development Theory.

Snaza, N., Appelbaum, P., Bayne, S., Carlson, D., Morris, M., Rotas, N., Sandlin, J., Wallin, J. \& Weaver, J. (2014). Toward a Posthumanist Education, Journal of Curriculum Theorizing, 30 (2), 39-55.

Sparkes, A.C. (2007). Embodiment, academics, and the audit culture: A story seeking consideration. Qualitative Research, 7(4), 521-550

Springgay, S. (2015). Approximate-Rigorous-Abstractions": Propositions of Activation for Posthumanist Research. In N. Snaza \& J. Weaver, (Editors). Posthumanism and Educational Research. (pp.76-88). New York, NY: Routledge.

St. Pierre, E. (2011). Post Qualitative Research: The Critique and the Coming After. En N. K. Denzin \& Y. S. Lincoln (eds.) The Sage Handbook of Qualitative Research (4a ed. Revisada), (pp. 611-625). Thousand Oaks: SAGE Publications Inc. 